\title{
Nickel-Catalyzed [4 + 2] Cycloaddition of Styrenes with Arynes via 1:1 Cross-Coupling: Synthesis of 9,10-Dihydrophenanthrenes
}

\author{
Teruhiko Kubo, Takeshi Fujita, and Junji Ichikawa* \\ Division of Chemistry, Faculty of Pure and Applied Sciences, University of Tsukuba, Tsukuba, Ibaraki 305-8571
}

(Received $<$ Month $><$ Date $>$, $<$ Year $>$; CL- $<$ No $>$; E-mail: $<$ insert corresponding e-mail address $>$ )

The $[4+2]$ cycloaddition of styrenes with arynes was achieved via 1:1 cross-coupling by a nickel catalyst. This protocol applies to a variety of styrenes and arynes generated in situ from $o$-(trimethylsilyl)aryl triflates to afford 9,10dihydrophenanthrenes involving substituted aromatic rings. By using this method, a naturally occurring stilbenoid is easily synthesized.

Arynes are attractive intermediates in synthetic organic chemistry. ${ }^{1}$ The strength of their application to organic synthesis is providing efficient routes for diverse and complex molecules, because their reactions enable forming two adjacent bonds in a one-pot operation, creating benzene-fused structures. Since Kobayashi and Sonoda developed a mild and convenient method for aryne generation using fluoride-induced 1,2-elimination of 2(trimethylsilyl)aryl triflates, ${ }^{2}$ aryne chemistry has drastically evolved. However, the high reactivities of arynes often cause introduction of multiple arynes. ${ }^{3,4}$ Recently, Biju and co-workers reported synthesis of 9-aryl-9,10-dihydrophenanthrenes via Diels-Alder [4 + 2] cycloaddition of styrenes with arynes, inevitably accompanied by the ene reaction with a second molecule of aryne (Scheme 1a). ${ }^{4 b}$ In this reaction, only styrenes bearing an electron-withdrawing group (e.g., cyano, trifluoromethyl, and ester) at the 4-position provide the 1:1 cycloadducts, 9,10-dihydrophenanthrenes bearing no substituent at the 9 and 10 -positions. ${ }^{5,6}$

(a) Previous Work by Biju

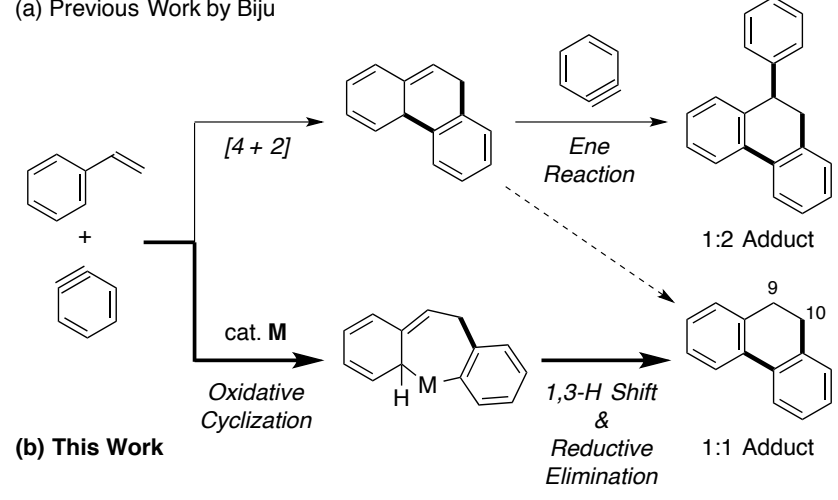

Scheme 1. Cycloaddition of styrenes and arynes.

To suppress multiple reactions, we assumed that transition metal catalysts can control the reactivity of arynes. When transition metal complexes are present, metal-mediated oxidative cyclization of styrenes with arynes proceeds as the initial step (Scheme 1b) instead of the Diels-Alder [4+2] cycloaddition. ${ }^{4-6}$ Subsequent 1,3-hydrogen shift followed by reductive elimination may afford 1:1 cycloadducts selectively. Based on this working hypothesis, we achieved an efficient nickel-catalyzed formal [4+ 2] cycloaddition of styrenes with arynes, ${ }^{7}$ enabling the synthesis of 9,10-dihydrophenanthrenes involving unsubstituted 9- and 10positions. The reactivity of arynes was adequately controlled using nickel, facilitating the one-to-one coupling of styrenes with arynes.

First, we investigated suitable conditions for nickelcatalyzed [ $4+2]$ cycloaddition using styrene (1a) and benzyne precursor 2a as model compounds in 1,4-dioxane (Table 1). When 1a was treated with $\mathbf{2 a}$ and $\mathrm{CsF}$ as a fluoride source for generating benzyne without any metal catalysts, the $[4+2]$ cycloadduct, 9,10-dihydrophenanthrene $\mathbf{3 a a}$ was obtained in only $3 \%$ yield (Entry 1). Under these conditions, the addition of a catalytic amount of $\mathrm{NiCl}_{2}$ afforded 3aa in 34\% yield along with the $[2+2]$ cycloadduct $4 \mathbf{a a}$, whereas the 1:2 adduct, 9-phenyl9,10-dihydrophenanthrene was completely absent (Entry 2). To improve the yield of $\mathbf{3 a a}$, ligands used for $\mathrm{NiCl}_{2}$ were screened (Entries 3-7). Among the ligands examined, $\mathrm{PCy}_{3}$ dramatically increased the yield of 3aa (Entry 6). Due to screening of $\mathrm{Ni}$ sources, $\mathrm{NiCl}_{2}$ was revealed as most efficient and selective (Entries 6, 8-12). Employing $\mathrm{NiCl}_{2}\left(\mathrm{PCy}_{3}\right)_{2}$ instead of $\mathrm{NiCl}_{2}$ and $\mathrm{PCy}_{3}$ separately, improved the yield of 3aa up to $65 \%$ (Entry 13 ). Finally, increasing the amount of 2a (1.5 equiv) afforded 3aa in $76 \%$ yield (Entries 14).

Table 1. Screening of conditions for cycloaddition of $\mathbf{1 a}$ with $2 \mathbf{a}$

\begin{tabular}{|c|c|c|c|c|}
\hline $1 a$ & $+{ }^{\mathrm{TfO}}$ & $\begin{array}{l}{[\mathrm{Ni} \text { (10 mol\%) }} \\
\text { gand (30 mol\% } \\
\text { CsF ( } 2.4 \text { equiv) } \\
\begin{array}{c}1,4-D i o x a n e \\
100^{\circ} \mathrm{C}, 16 \mathrm{~h}\end{array}\end{array}$ & 3aa & 4aa \\
\hline Entry & {$[\mathrm{Ni}]$} & Ligand & 3aa $(\%)^{a}$ & 4aa $(\%)^{a}$ \\
\hline 1 & - & - & 3 & N.D. ${ }^{b}$ \\
\hline 2 & $\mathrm{NiCl}_{2}$ & - & 34 & 3 \\
\hline 3 & $\mathrm{NiCl}_{2}$ & $\mathrm{PPh}_{3}$ & N.D. ${ }^{b}$ & N.D. ${ }^{b}$ \\
\hline 4 & $\mathrm{NiCl}_{2}$ & $\mathrm{PMe}_{3}$ & 46 & 7 \\
\hline 5 & $\mathrm{NiCl}_{2}$ & $\mathrm{P}(t-\mathrm{Bu})_{3}$ & 56 & 8 \\
\hline 6 & $\mathrm{NiCl}_{2}$ & $\mathrm{PCy}_{3}$ & 59 & 10 \\
\hline $7^{c}$ & $\mathrm{NiCl}_{2}$ & $\mathrm{IPr} \cdot \mathrm{HCl}$ & 52 & 9 \\
\hline 8 & $\mathrm{NiF}_{2}$ & $\mathrm{PCy}_{3}$ & 47 & 8 \\
\hline 9 & $\mathrm{NiBr}_{2}$ & $\mathrm{PCy}_{3}$ & 55 & 9 \\
\hline 10 & $\mathrm{Ni}(\mathrm{OAc})_{2}$ & $\mathrm{PCy}_{3}$ & 47 & 7 \\
\hline 11 & $\mathrm{Ni}(\mathrm{acac})_{2}$ & $\mathrm{PCy}_{3}$ & 17 & 3 \\
\hline 12 & $\mathrm{Ni}(\operatorname{cod})_{2}$ & $\mathrm{PCy}_{3}$ & 56 & 15 \\
\hline 13 & $\mathrm{NiCl}_{2}\left(\mathrm{PCy}_{3}\right)_{2}$ & - & 65 & 5 \\
\hline $14^{d}$ & $\mathrm{NiCl}_{2}\left(\mathrm{PCy}_{3}\right)_{2}$ & - & 76 & 10 \\
\hline
\end{tabular}

a Yield was determined by ${ }^{1} \mathrm{H}$ NMR spectroscopy using 1,1,2,2tetrachloroethane as an internal standard. ${ }^{b}$ N.D. $=$ Not detected. ${ }^{c} \mathrm{IPr} \cdot \mathrm{HCl}$ 
(20 mol\%) and $\mathrm{K}_{2} \mathrm{CO}_{3}(20 \mathrm{~mol} \%)$ were used. ${ }^{d}$ 2a (1.5 equiv) and $\mathrm{CsF}$ (3.0 equiv) were used.

The scope of the reaction related to substituted styrenes 1 was explored under optimal conditions using $\mathbf{2 a}$ (Table 2). Nonsubstituted and 4'-phenylated styrenes $\mathbf{1 a}$ and $\mathbf{1 b}$ underwent nickel-catalyzed $[4+2]$ cycloaddition with $\mathbf{2 a}$ to afford the corresponding 9,10-dihydrophenanthrenes 3aa and $\mathbf{3 b a}$ in isolated $67 \%$ and $70 \%$ yields, respectively, after purification by gel permeation chromatography (GPC). Reactions of styrenes 1c and 1d bearing electron-donating methyl and methoxy groups at the para positions proceeded fairly, whereas utilizing electronwithdrawing acetyl- and trifluoromethyl-bearing styrenes $\mathbf{1 e}$ and 1f produced rather high yields. Since boryl and halogen substituents like pinacolboryl, fluorine, chlorine, and bromine were tolerated under the reaction conditions, dihydrophenanthrenes 3ga-3ka bearing boryl and halogen substituents on the benzene rings were synthesized in high yields. A sterically demanding bromine substituent at the ortho position caused no negative effect on the reaction. Cycloaddition of $\alpha$ substituted styrenes were also investigated under the same conditions. The $\alpha$-alkylated and $\alpha$-arylated styrenes $\mathbf{1 l}$ and $\mathbf{1 m}$ were applicable to the reaction to afford dihydrophenanthrenes 3la and 3ma, respectively.

Table 2. Nickel-catalyzed [4 +2$]$ cycloaddition of substituted styrenes $\mathbf{1}$ with aryne precursor $\mathbf{2 a}^{a}$

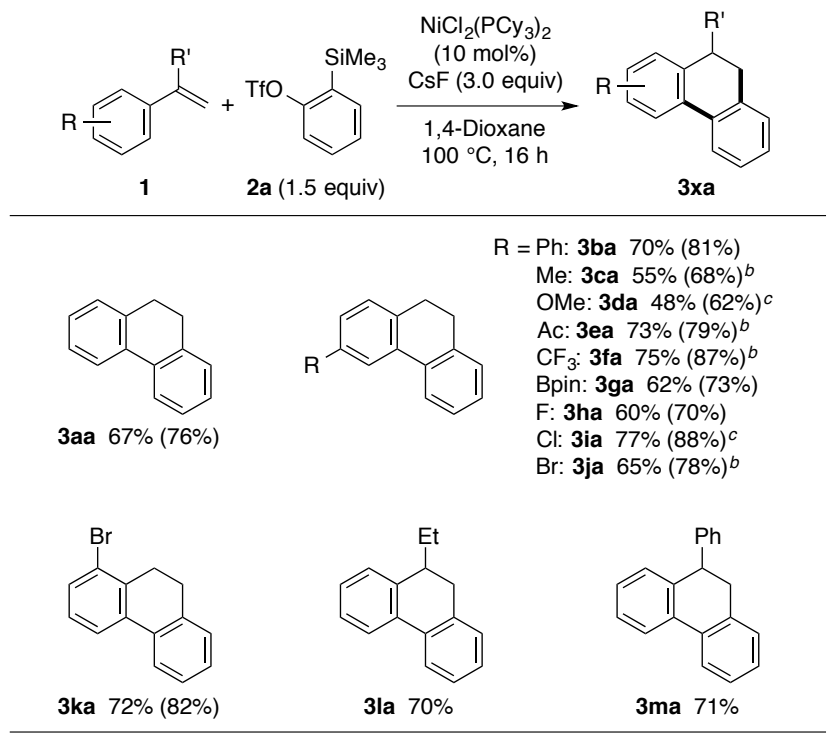

${ }^{a}$ Isolated yield after purification by preparative GPC. Yield determined by ${ }^{1} \mathrm{H}$ NMR spectroscopy using 1,1,2,2-tetrachloroethane as an internal standard is given in parentheses. ${ }^{b}$ 2a (2.0 equiv) and $\mathrm{CsF}$ (4.0 equiv) were used. ${ }^{c} \mathrm{NiCl}_{2}\left(\mathrm{PCy}_{3}\right)_{2}(15 \mathrm{~mol} \%)$ was used.

Substituted aryne precursors $\mathbf{2}$ were also examined in reactions using styrene (1a) under the optimal conditions (Table 3 ). Reactions of $\mathbf{1 a}$ with aryne precursors $\mathbf{2 b}-\mathbf{2} \mathbf{d}$ bearing two methyl, methoxy, and fluorine substituents proceeded to afford corresponding 2,3-disubstituted 9,10-dihydrophenanthrenes 3ab3ad in high yields. Aryne precursor $2 \mathrm{e}$ with a naphthalene ring formed tetracyclic dihydrotetraphene 3ae in $46 \%$ isolated yield.

Table 3. Nickel-catalyzed $[4+2]$ cycloaddition of styrene (1a) with substituted aryne precursors $\mathbf{2}^{a}$

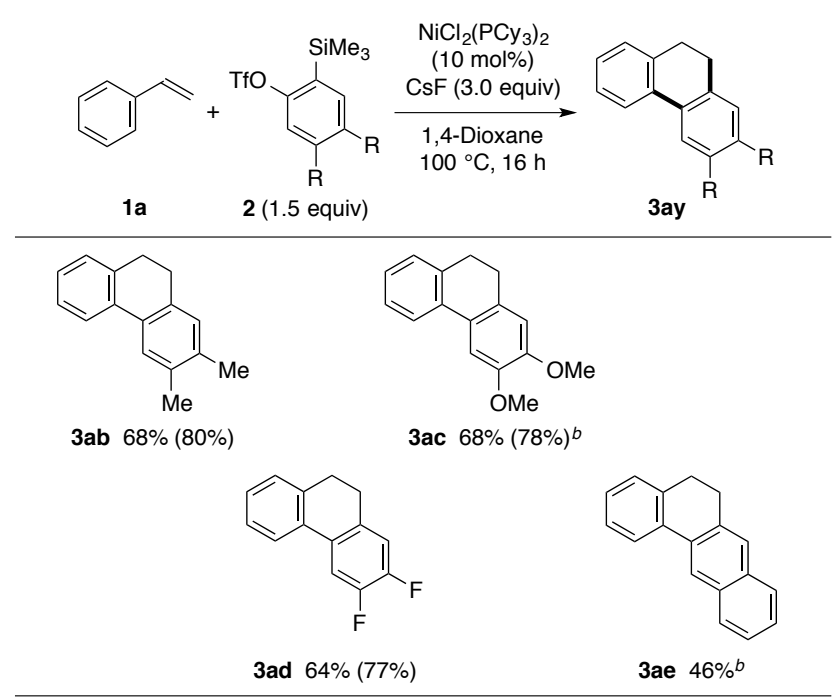

${ }^{a}$ Isolated yield after purification by preparative GPC. Yield determined by ${ }^{1} \mathrm{H}$ NMR spectroscopy using 1,1,2,2-tetrachloroethane as an internal standard is given in parentheses. ${ }^{b} \mathbf{2}$ (2.0 equiv) and $\mathrm{CsF}$ (4.0 equiv) were used.

This protocol was applied for the synthesis of a natural product. Callosumin (3nc) $)^{8,9}$ a naturally occurring stilbenoid ${ }^{10}$ was synthesized in $37 \%$ isolated yield via the nickel-catalyzed [ 4 +2 ] cycloaddition using 3,5-dimethoxystyrene (1n) and aryne precursor 2c (Scheme 2).

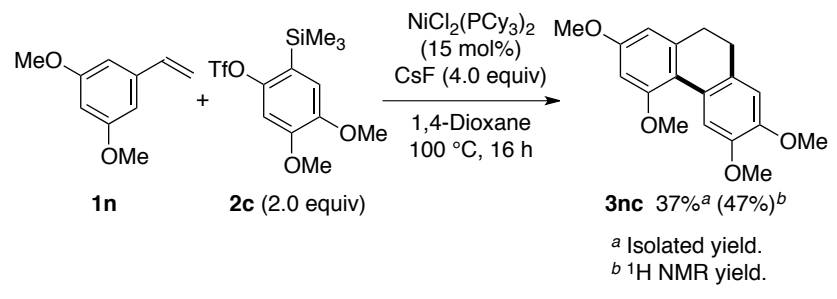

Scheme 2. Synthesis of callosumin (3nc).

Due to the formation of the [2+2] cycloadducts 4 and 9,10dihydrophenanthrenes $\mathbf{3}$, we propose the reaction mechanism in Scheme 3 . The reaction begins with coordination of styrenes 1 and arynes generated from $\mathbf{2}$ to in-situ generated $\mathrm{Ni}(0)$ species. ${ }^{11}$ Two ways exist for subsequent nickel-mediated oxidative cyclization between $\mathbf{1}$ and arynes to produce: (i) seven-membered nickelacycles A and (ii) five-membered nickelacycles B. Styrenes $\mathbf{1}$ react as 1,3-dienes via dearomatization to form $\mathbf{A}$, whereas the vinylic moieties of $\mathbf{1}$ only are involved in the formation of $\mathbf{B}$. We speculate that by-products $\mathbf{4}$ are formed via reductive elimination from $\mathbf{B}$. In addition, nickelacycles $\mathbf{A}$ are formed even from $\mathbf{B}$ via ring expansion. Rearomatizing 1,3-hydrogen shift in $\mathbf{A}$ followed by reductive elimination affords 9,10-dihydrophenanthrenes $\mathbf{3}$ along with catalytically active $\mathrm{Ni}(0)$ species. 


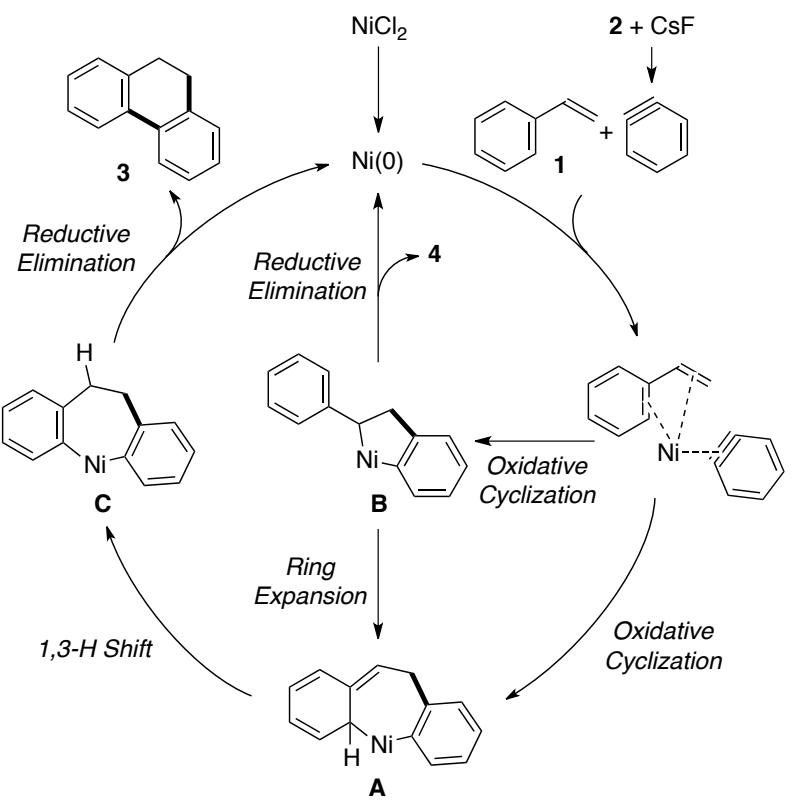

Scheme 3. Plausible reaction mechanism.

In summary, nickel-catalyzed [4 +2$]$ cycloaddition of styrenes with arynes in a 1:1 ratio was accomplished. Consequently, we controlled the reactivity of arynes using a nickel catalyst. Although 9,10-dihydrophenanthrenes with no substituent at the 9- or 10-positions are vital as naturally occurring stilbenoids in pharmaceutical and agrochemical sciences, conventional approaches for their production often exhibit poor efficiency/selectivity ${ }^{12}$ or narrow substrate scope. ${ }^{13}$ These limitations were eliminated in this study by introducing an efficient method for the synthesis of 9,10-dihydrophenanthrenes.

This work was financially supported by JSPS KAKENHI Grant Number JP19H02707 (J.I.) in Grant-in-Aid for Scientific Research (B), JSPS KAKENHI Grant Number JP18H04234 (J.I.) in Precisely Designed Catalysts with Customized Scaffolding, and JSPS KAKENHI Grant Number JP18K05116 (T.F.) in Grant-in-Aid for Scientific Research (C).

Supporting Information is also available electronically on the CSJ-Journal Web site, http://www.csj.jp/journals/chem-lett/index.html.

\section{References and Notes}

1 For recent reviews on arynes, see: a) S. Yoshida, T. Hosoya, Chem. Lett. 2015, 44, 1450-1460. b) R. Karmakar, D. Lee, Chem. Soc Rev. 2016, 45, 4459-4470. c) Y. Zeng, J. Hu, Synthesis 2016, 48, 2137-2150. d) S. S. Bhojgude, A. Bhunia, A. T. Biju, Acc. Chem. Res. 2016, 49, 1658-1670. e) J.-A. Garcia-López, M. F. Greaney, Chem. Soc. Rev. 2016, 45, 6766-6798. f) J. Shi, Y. Li, Y. Li, Chem. Soc. Rev. 2017, 46, 1707-1719. g) F. I. M. Idiris, C. R. Jones, Org. Biomol. Chem. 2017, 15, 9044. h) R. A. Dhokale, S. B. Mhaske, Synthesis 2018, 50, 1-16. i) T. Roy, A. T. Biju, Chem. Commun. 2018, 54, 2580-2594. j) A. Yoshimura, A. Saito, V. V. Zhdankin, Chem. Eur. J. 2018, 24, 15156-15166. k) H. Takikawa, A. Nishii, T. Sakai, K. Suzuki, Chem. Soc. Rev. 2018, 47, 8030-8056.

2 Y. Himeshima, T. Sonoda, H. Kobayashi, Chem. Lett. 1983, 12, 1211-1214.

3 a) J. M. Brinkley, L. Friedman, Tetrahedron Lett. 1972, 13, 4141. b) I. Tabushi, H. Yamada, Z. Yoshida, R. Oda, Bull. Chem. Soc. Jpn. 1977, 50, 285-290. c) J.-C. Hsieh, D. K. Rayabarapu, C.-H. Cheng, Chem. Commun. 2004, 532-533. d) I. Quintana, A. J.
Boersma, D. Peña, D. Pérez, E. Guitián, Org. Lett. 2006, 8, 33473349. e) H. Yoshida, T. Morishita, H. Nakata, J. Ohshita, Org. Lett. 2009, 11, 373-376. f) N. Saito, K. Shiotani, A. Kimbara, Y. Sato, Chem. Commun. 2009, 4284-4286. g) T. Morishita, H. Yoshida, J. Ohshita, Chem. Commun. 2010, 46, 640-642. h) M.-J. OlivaMadrid, I. Saura-Llamas, D. Bautista, J. Vicente, Chem. Commun. 2013, 49, 7997-7999. i) K. Neog, D. Dutta, B. Das, P. Gogoi, Org. Lett. 2017, 19, 730-733.

4 a) W. L. Dilling, Tetrahedron Lett. 1966, 9, 939-941. b) S. S. Bhojgude, A. Bhunia, R. G. Gonnade, A. T. Biju, Org. Lett. 2014, 16, 676-679. c) Z. Chen, X. Han, J.-H. Liang, J. Yin, G.-A. Yu, S.H. Liu, Chin. Chem. Lett. 2014, 25, 1535-1539.

5 For an intramolecular reaction, see: J. C. Estévez, R. J. Estévez, L. Castedo, Tetrahedron Lett. 1992, 33, 6883-6884.

6 An intermolecular reaction with a 1,4-benzodiyne precursor in a 1:1 manner was achieved albeit with low efficiency. See: I. Pozo, Z Majzik, N. Pavlicek, M. Melle-Franco, E. Guitián, D. Peña, L. Gross, D. Pérez, J. Am. Chem. Soc. 2019, 141, 15488-15493.

7 For seclected reports on nickel-catalyzed cyclization with arynes, see: a) J.-C. Hsieh, C.-H. Cheng, Chem. Commun. 2005, 2459 2461. b) J.-C. Hsieh, C.-H. Cheng, Chem. Commun. 2008, 2992 2994. c) N. Saito, K. Shiotani, A. Kinbara, Y. Sato, Chem. Commun. 2009, 4284-4286. d) Z. Qiu, Z. Xie, Angew. Chem. Int. Ed. 2009, 48, 5729-5732. e) D. A. Candito, M. Lautens, Synlett 2011, 1987-1992. f) V. H. Thorat, N. S. Upadhyay, M. Murakami, C.-H. Cheng, Adv. Synth. Catal. 2018, 360, 284-289.

8 For the synthesis of callosumin, see: R. M. Letcher, L. R. M. Nhamo, J. Chem. Soc. C 1971, 3070-3076.

9 For the isolation of callosumin from plants, see: a) P. L. Majumder, S. Baneriee, S. Sen, Phytochemistry 1996, 42, 847-852. b) H. Anton, L. Kraut, R. Mues, M. I. Morales Z. Phytochemistry 1997, 46, 1069-1075. c) P. L. Majumder, S. Majumder, S. Sen, Phytochemistry 2003, 62, 591-596.

10 For selected reviews on stilbenoids, see: a) I. J. Flores-Sanchez, R. Verpoorte, Phytochem. Rev. 2008, 7, 615-639. b) T. Shen, X.-N. Wang, H.-X. Lou, Nat. Prod. Rep. 2009, 26, 916-935. c) C. Rivière, A. D. Pawlus, J.-M. Mérillon, Nat. Prod. Rep. 2012, 29, 1317-1333. d) M. Dvorakova, P. Landa, Pharmacol. Res. 2017, 124, 126-145. e) B. C. Akinwumi, K.-A. M. Bordun, H. D. Anderson, Int. J. Mol. Sci. 2018, 19, 792.

11 A similar method for aryne generation can generate low-valent active $\mathrm{Pd}$ species from high-valent complexes even under reductant-free conditions. See: M. Feng, B. Tang, N. Wang, H.-X. Xu, X. Jiang, Angew. Chem. Int. Ed. 2015, 54, 14960-14964.

12 For recent reports via reduction, see: a) E. Schachtl, J. S. Yoo, O. Y Guitiérrez, F. Studt, J. A. Lercher, J. Catal. 2017, 352, 171-181. b) A. J. Smith, A. Young, S. Rohrbach, E. F. O'Connor, M. Allison, H.-S. Wang, D. L. Poole, T. Tuttle, J. A. Murphy, Angew. Chem. Int. Ed. 2017, 56, 13747-13751. c) W. Luo, H. Shi, E. Schachtl, O. Y. Gutiérrez, J. A. Lercher, Angew. Chem. Int. Ed. 2018, 57, 14555-14559.

13 For recent reports via dimerization, see: a) D. Toummini, F. Ouazzani, M. Taillefer, Org. Lett. 2013, 15, 4690-4693. b) G. Revol, T. McCallum, M. Morin, F. Gagosz, L. Barriault, Angew. Chem. Int. Ed. 2013, 52, 13342-13345. c) Y. Liu, J. Bergès, Y. Zaid, F. O. Chahdi, A. Van Der Lee, D. Harakat, E. Clot, F. Jaroschik, M. Taillefer, J. Org. Chem. 2019, 84, 4413-4420 
The diagram is acceptable in a colored form. Publication of the colored G.A. is free of charge.

For publication, electronic data of the colored G.A. should be submitted. Preferred data format is EPS, PS, CDX, PPT, and TIFF. If the data of your G.A. is "bit-mapped image" data (not "vector data"), note that its print-resolution should be 300 dpi.

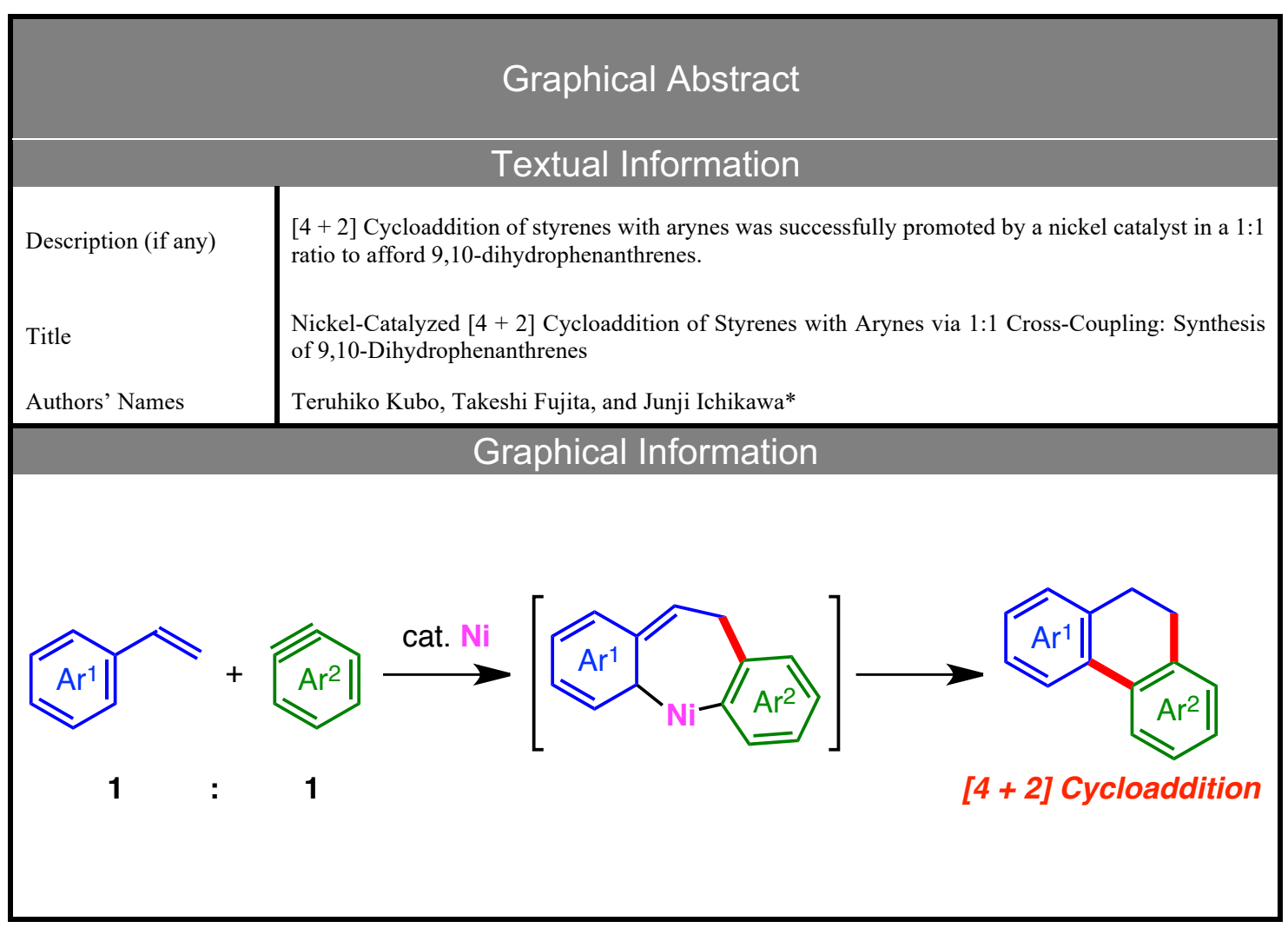

